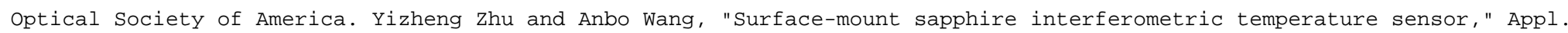
Opt.45,6071-6076(2006). doi: 10.1364/AO.45.006071

\title{
Surface-mount sapphire interferometric temperature sensor
}

\author{
Yizheng Zhu and Anbo Wang
}

\begin{abstract}
A fiber-optic high-temperature sensor is demonstrated by bonding a $45^{\circ}$-polished single-crystal sapphire fiber on the surface of a sapphire wafer, whose optical thickness is temperature dependent and measured by white-light interferometry. A novel adhesive-free coupling between the silica and sapphire fibers is achieved by fusion splicing, and its performance is characterized. The sensor's interference signal is investigated for its dependence on angular alignment between the fiber and the wafer. A prototype sensor is tested to $1170^{\circ} \mathrm{C}$ with a resolution of $0.4^{\circ} \mathrm{C}$, demonstrating excellent potential for high-temperature measurement. (C) 2006 Optical Society of America

OCIS codes: $\quad 060.2370,120.6780$.
\end{abstract}

\section{Introduction}

Temperature sensing is a key topic in fiber-optic sensor research. Numerous schemes have been proposed since the early 1980 s for a variety of applications from biological monitoring to industrial sensing. For high temperatures well above $1000^{\circ} \mathrm{C}$, single-crystal optical fibers have been widely adopted for sensor fabrication owing to their excellent mechanical and optical properties at these elevated temperatures. Most of the fiber-optic high-temperature sensors can be categorized into three main techniques: thermal radiation, fluorescence lifetime, and optical interference. The simplest form of radiation-based sensor is a fiber version of an optical pyrometer, often referred to as a lightpipe radiation thermometer, a device used in the semiconductor industry for wafer temperature monitoring during rapid thermal processing by collecting radiation from the target surface and transmitting it to the detector. ${ }^{1}$ Temperature can then be inferred using the standard two-color approach. Other than merely a light collecting and transmitting pipe, another type of radiation-based sensor generates its own radiation signal with a metalcoated sapphire fiber tip that forms a blackbody

Yizheng Zhu (yizhul@vt.edu) and Anbo Wang are with the Center for Photonics Technology, Department of Electrical and Computer Engineering, Virginia Polytechnic Institute and State University, Blacksburg, Virginia 24061.

Received 1 November 2005; revised 22 February 2006; accepted 4 March 2006; posted 5 May 2006 (Doc. ID 65736).

0003-6935/06/246071-06\$15.00/0

(C) 2006 Optical Society of America cavity. ${ }^{2,3}$ Operation up to $2300{ }^{\circ} \mathrm{C}$ has been demonstrated using zirconia single-crystal fiber. ${ }^{4}$ In both types, the radiation emitted by the fiber lightpipe itself adds to the target radiation and thus constitutes an error source, leaving the measurement sensitive to environmental temperature changes around the lightpipe.1,5,6 Therefore in situ calibration is usually required and brings inconvenience to its field application. In a fluorescence-based sensor, fiber is doped and the dopant is excited by laser pulses to generate fluorescence, whose decay rate is temperature dependent and used as the measurand. Operating temperature of $1600{ }^{\circ} \mathrm{C}$ has been achieved by a Yb-doped single-crystal YAG optical fiber. ${ }^{7}$ The temperature dependence of the decay rate usually exhibits a vastly changing profile, increasing slowly at low temperature and very quickly toward the hightemperature end.7-9 This will leave the sensor with quite different sensitivities and performance at difference temperature ranges and therefore may limit its dynamic range. As another widely employed technique, fiber-optic interferometric sensors are known for their large dynamic range, high resolution, and high accuracy. Various principles have been proposed based on both instrinsic and extrinsic Fabry-Perot interfereometers. ${ }^{10-12}$ However, one major concern of these sensors in high-temperature measurements is the large modal volume of single-crystal sapphire fibers. The intermodal dispersion makes it difficult to generate good fringes that are sensitive to the quality and the parallelism of the interferometer surfaces..$^{13,14}$

To address this issue, the authors have previously reported a sapphire extrinsic Fabry-Perot interferometric sensor, using a sapphire wafer as the inter- 


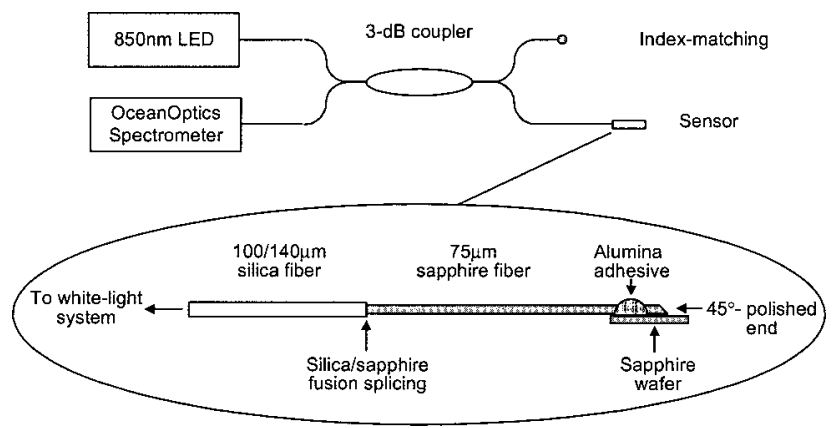

Fig. 1. System schematic.

ferometer. ${ }^{15}$ The high quality and parallelism of the wafer surfaces allow easy generation of good interference fringes even for highly multimode sapphire fiber. As the measurand, the wafer's optical thickness is temperature dependent as both the refractive index and the physical thickness increase with temperature. In this paper, we propose another simpler and smaller configuration by $45^{\circ}$ polishing the fiber end and placing the wafer to the side of the fiber. In addition to high temperature test, also discussed are the signal's dependence on fiber-wafer alignment and a novel silica-sapphire fiber coupling method. Unlike most other fiber-optic sensors, this surface-mount structure aligns the fiber in the wafer plane, which is not only suitable for temperature measurement, but also opens possibilities for other high-temperature surface measurement such as strain.

\section{System Setup}

Figure 1 shows the schematic of the white-light interferometric system. Broadband light from an $850 \mathrm{~nm}$ light-emitting diode (LED) travels to the sensor through a 100/140 $\mu \mathrm{m}$ multimode fiber $3 \mathrm{~dB}$ coupler. The reflected signal carries the wafer's interference spectrum to be detected by an OceanOptics USB2000 miniature spectrometer. In the sensing arm, a $75 \mu \mathrm{m}$ diameter sapphire fiber (MicroMaterials, Inc.) is $45^{\circ}$ end-polished and bonded by high-temperature adhesive on a $59 \mu \mathrm{m}$ thick C-plane sapphire wafer of a manually cut $1 \mathrm{~mm} \times 2 \mathrm{~mm}$ size. The other end of the sapphire fiber is spliced to the $100 / 140 \mu \mathrm{m}$ silica fiber. The angled fiber end bends the incoming light toward the wafer and redirects the reflected signal from the wafer back into the system. This arrangement aligns the fiber in the plane of the wafer, making a direct bond possible without the need for extra supporting structure. Therefore it minimizes the sensor weight and size. Submillimeter size is achievable if the wafer is machine diced.

\section{Signal Processing}

Figure 2(a) shows an enlarged view of the sensor head. At $850 \mathrm{~nm}$, sapphire has refractive indices around 1.76 for both the ordinary and the extraordinary trays, translating to a $34.6^{\circ}$ critical angle for total internal reflection. Different guided modes in the sapphire fiber have different incident angles at

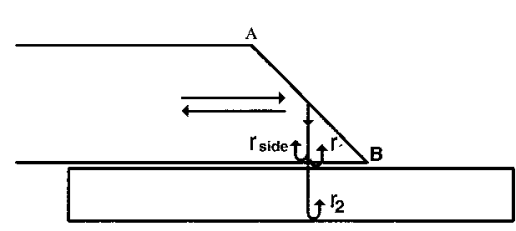

(a)

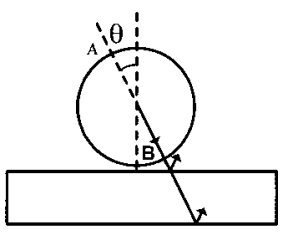

(b)
Fig. 2. Sensor head structure.

the polished surface, but all within a certain range around $45^{\circ}$. Given that $34.6^{\circ}$ is far less than $45^{\circ}$, the majority of the incoming modes and energy is expected to be bent toward the wafer by the $45^{\circ}$ polished end. This is supported by experiments where visible light is injected into the sapphire fiber, and at the polished end it can be observed that light is bent $90^{\circ}$ by the polished surface and comes out of the fiber perpendicular to it with a certain divergence angle. From other directions, there is practically no light observed. The bent light then travels through the fiber's curved side surface and the two parallel surfaces of the wafer and generates three reflections back into the system. The signal received at the spectrometer can be written as

$$
\begin{aligned}
I_{\text {total }}(\lambda)= & I_{\mathrm{D}}(\lambda)+I_{\mathrm{B}}(\lambda) \\
& +I_{\mathrm{LED}}(\lambda)\left(r_{\text {couple }}+r_{\text {side }}\right)+I_{\mathrm{LED}}(\lambda) \\
& \times\left[r_{1}+r_{2}-2 V \sqrt{r_{1} r_{2}} \cos \frac{4 n(T) d(T) \pi}{\lambda}\right],
\end{aligned}
$$

where $I_{\mathrm{D}}(\lambda), I_{\mathrm{B}}(\lambda)$, and $I_{\mathrm{LED}}(\lambda)$ are the intensities of the dark current of the spectrometer, the blackbody radiation, and the LED, respectively; $r_{\text {couple }}, r_{\text {side }}, r_{1}$, and $r_{2}$ are reflectance at interfaces along the path (the silica-sapphire fiber coupling point, the side of the sapphire fiber, and the first and second surfaces of the sapphire wafer, respectively); $n$ is the ordinaryray refractive index of sapphire since the wafer is C-plane, $d$ is the wafer thickness, $V$ is the fringe visibility, $\lambda$ is the wavelength, and $T$ is the temperature. Figure 3 shows a typical sensor spectrum at room temperature, whose interference is very clear as compared to that of a polished fiber end without the wafer.

Equation (1) only includes the interference between $r_{1}$ and $r_{2}$, not those between $r_{\text {side }}$ and $r_{1}$ or $r_{2}$. The reason is that the fiber side is so curved and unparallel to the wafer surfaces that it is practically impossible for $r_{\text {side }}$ to interfere with $r_{1}$ or $r_{2}$. This also demonstrates why high surface parallelism and quality are critical for fringe generation in multimode fibers.

Signal processing is based on the fact that all terms in Eq. (1) except the cosine are low-frequency components, and therefore in Fourier transform the high-frequency cosine term can be filtered out by a bandpass filter, giving ${ }^{15}$

$$
2 V \sqrt{r_{s 1} r_{s 2}} I_{\mathrm{LED}}(\lambda) \cos \frac{4 n(T) d(T) \pi}{\lambda} .
$$




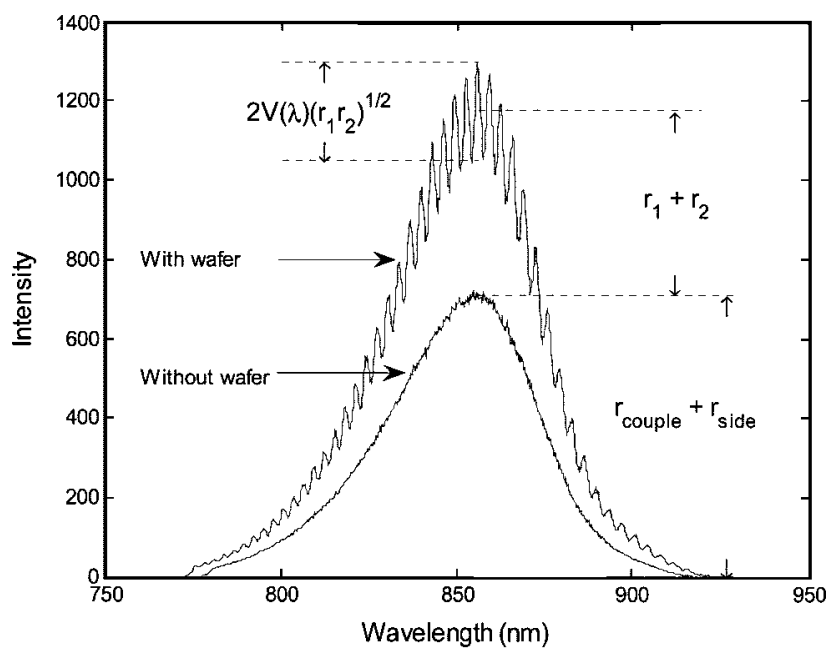

Fig. 3. Sensor spectrum.

This term can be further normalized by Hilbert transform to result in

$$
\cos \frac{4 n(T) d(T) \pi}{\lambda}
$$

from which the wafer's optical thickness, $n(T) d(T)$, can be determined by the positions of interference valleys and/or peaks.

\section{Sensor Fabrication}

It is clear in Fig. 3 that the wafer fringes add on top of a large background raised by several sources. During the fabrication, it is important to have sufficient fringe amplitude for a good signal-to-noise ratio. However, when the fiber rotates, the incident light will not be at right angle to the wafer and the backcoupling efficiency will decrease for the returned signals, as illustrated in Fig. 2(b). Figure 4 (a) plots the diminishing spectra of the signals reflected from the wafer,
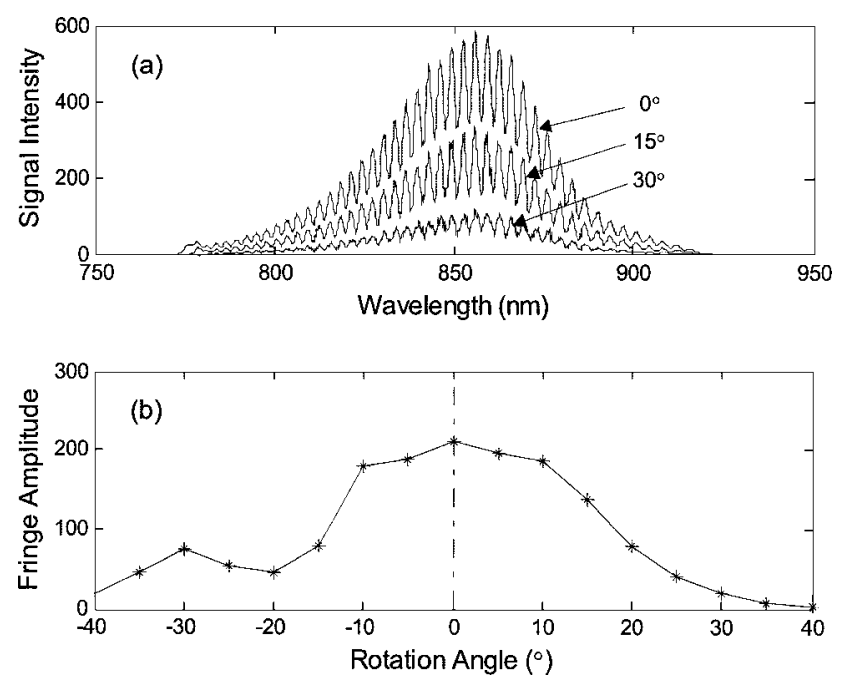

Fig. 4. Angular dependence of signal intensity and fringe amplitude.

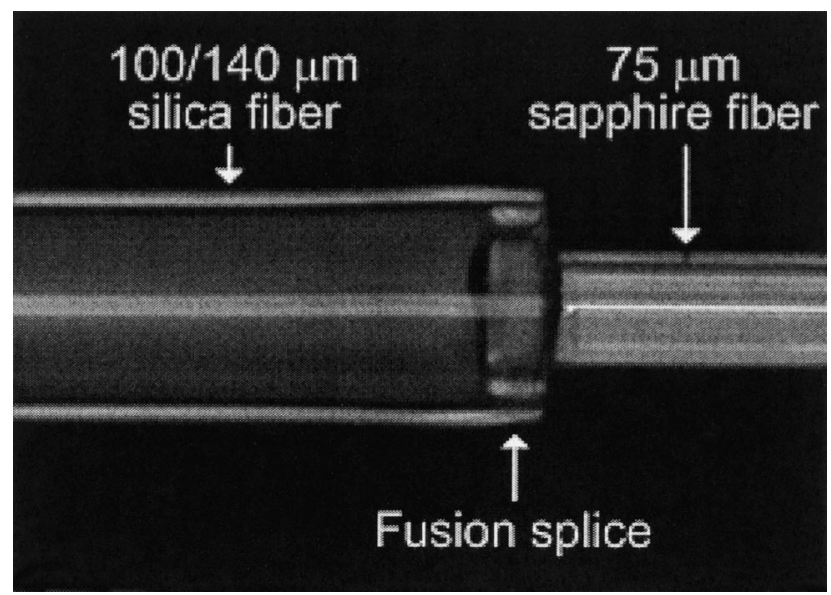

Fig. 5. Fusion splice of silica fiber and sapphire fiber.

$$
I_{\mathrm{LED}}(\lambda)\left[r_{1}+r_{2}-2 V \sqrt{r_{1} r_{2}} \cos \frac{4 n(T) d(T) \pi}{\lambda}\right]
$$

at increasing angles. Also plotted is the normalized fringe intensity, $2 V \sqrt{r_{s 1} r_{s 2}}$, against the rotating angle $\theta$ [Fig. 4(b)]. It can be seen that the fringe intensity remains at similar levels within a rather wide angle of about $\pm 15^{\circ}$, offering good fabrication tolerance for this angular misalignment. The irregularities and asymmetry of the curve is due to the hexagonal shape of single-crystal sapphire fiber, which makes the polished end shape dependent on the polishing direction.

The coupling of the silica and sapphire fibers was made through arc fusion splicing. Different from a previously reported splice using aluminosilicate glass as the interlayer, ${ }^{11}$ it is adhesive-free because the 100 $\mu \mathrm{m}$ Ge-doped core of the silica fiber softens at a much lower temperature than the undoped cladding. Under properly reduced arc duration and power, the core will be softened, while the cladding, as well as the sapphire fiber, will remain almost intact. Prior to the arc, the sapphire fiber is brought to the close proximity of the silica fiber. It is then heated by the arc and expands, protruding into the melted core of the silica fiber to make the connection. Multiple arcs may be necessary, depending on the power and duration.

A photo of such a splice is shown in Fig. 5. Figure 6 plots the reflected power variation against the number of arcs. This power consists of both the reflection from the coupling point, $r_{\text {couple }}$, and the reflected signal from the sapphire fiber end, $r_{\text {signal. }}$ The power tends to first decrease and then stabilize with the number of arcs. In Fig. 6, a total of $5 \mathrm{~dB}$ loss was recorded from the first arc to the last. However, much of this loss is attributed to the decrease of $r_{\text {couple }}$ because of the incomplete contact at the silica-sapphire interface, which may leave small high-reflecting air voids during the first several arcs, as a result of low arc power and duration. As more arcs are applied, the two fibers come to better physical contact to reduce the high-reflecting voids and the optical power also reduces. Since $r_{\text {couple }}$ can be removed in signal processing, its value is not critical to the application. The loss 


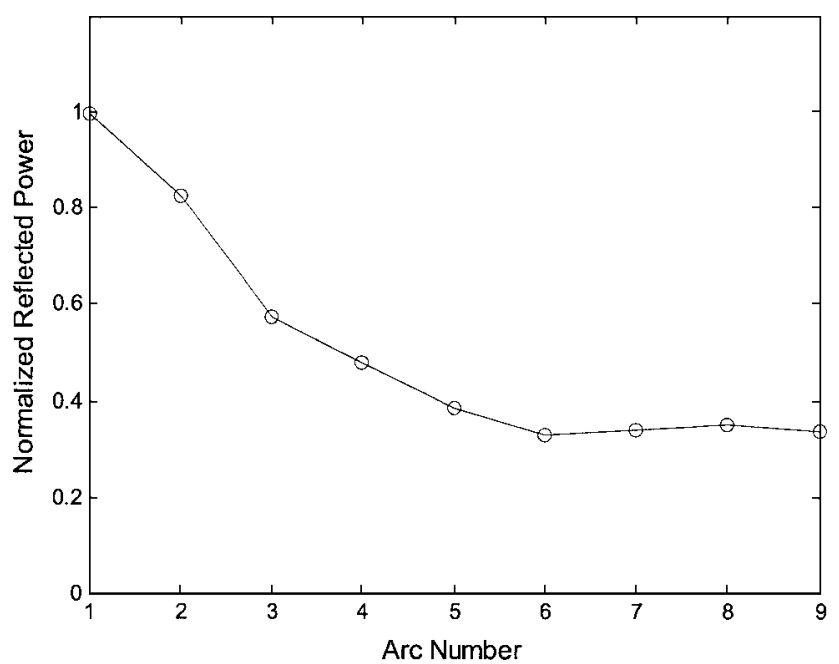

Fig. 6. Reflected power versus number of arc.

of $r_{\text {signal }}$ is, however, of more importance because it will determine the signal intensity. $r_{\text {signal }}$ is measured by immersing the sapphire fiber end from air into an index matching oil $(n=1.522)$ and recording the intensity change that is proportional to $r_{\text {signal. }}$. By comparing $r_{\text {signal }}$ before and after the splice, power loss as low as $0.5 \mathrm{~dB}$ has been measured. It is possibly due to the deformation of the $100 / 140 \mu \mathrm{m}$ fiber, which may scatter the light out of the coupling point. This method avoids any adhesive, producing a simple and robust joint. It is also successful splicing $100 \mu \mathrm{m}$ diameter sapphire fibers but fails for larger ones due to the apparent silica fiber core size limitation.

\section{Temperature Test and Analysis}

\section{A. Temperature Response}

The sensor's temperature response was tested in a furnace (Thermolyne 48000, maximum temperature $1200{ }^{\circ} \mathrm{C}$ ) from $24{ }^{\circ} \mathrm{C}$ to $1170{ }^{\circ} \mathrm{C}$. An S-type thermocouple was used for a temperature reference. Figure 7 plots the change of optical thickness (open circles)

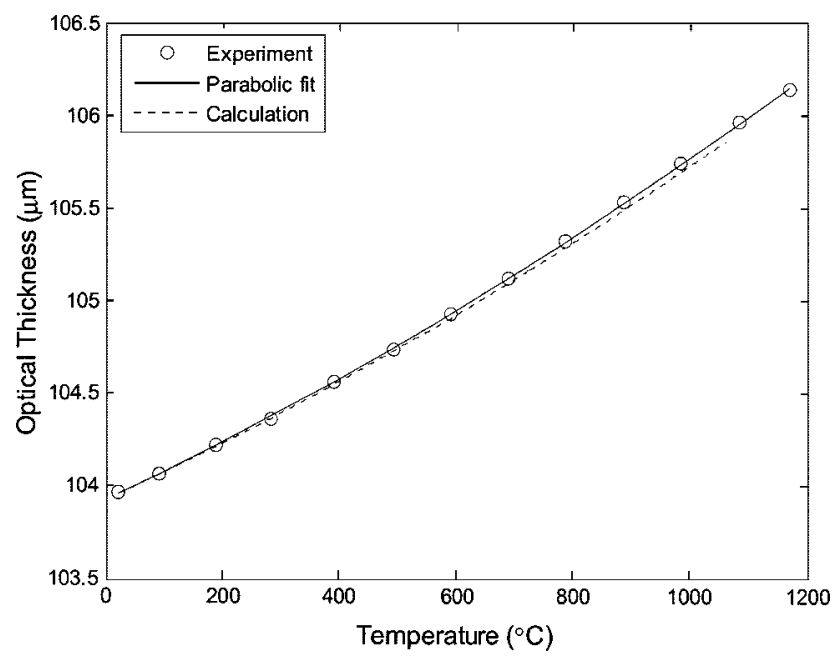

Fig. 7. Temperature response. with respect to temperature measured by the S-type thermocouple. The data are fitted by a parabola as the following,

$$
\begin{aligned}
\mathrm{OT}= & n(T) d(T) \\
= & 103.913+1.507 \times 10^{-3} T+3.484 \\
& \times 10^{-7} T^{2}(\mu \mathrm{m}),
\end{aligned}
$$

and shown in Fig. 7 (solid curve). Also plotted is the calculated temperature response (dotted line) from published data of sapphire's thermal expansion and its refractive index. The recommended values of $C$-axis thermal expansion of sapphire can be written as $^{16}$

$$
\begin{aligned}
d(T)= & \left(1-0.192+5.927 \times 10^{-4} T+2.142 \times 10^{-7} T^{2}\right. \\
& \left.-2.207 \times 10^{-11} T^{3}\right) d_{0},
\end{aligned}
$$

where $T$ is the absolute temperature in Kelvin, $d(T)$ is the thickness at $T$, and $d_{0}$ is the initial thickness at $293 \mathrm{~K}\left(20^{\circ} \mathrm{C}\right)$.

For the temperature dependence of the refractive index of the ordinary ray in sapphire, $n(T)$, Tapping et al. have measured this value from $24^{\circ} \mathrm{C}$ to $1060{ }^{\circ} \mathrm{C}$ for wavelengths of $633 \mathrm{~nm}$ and $799 \mathrm{~nm},{ }^{17}$ as given by

$$
\begin{aligned}
n(T)_{633 \mathrm{~nm}}= & 1.76565+1.258 \times 10^{-5} \mathrm{~T}+4.06 \\
& \times 10^{-9} T^{2}, \\
n(T)_{799 \mathrm{~nm}}= & 1.75991+1.229 \times 10^{-5} \mathrm{~T}+3.10 \\
& \times 10^{-9} T^{2},
\end{aligned}
$$

where $T$ is temperature in degrees Celsius. By linear extrapolation from these two equations, a good estimation of $n(T)$ for $850 \mathrm{~nm}$ can be obtained as the following:

$$
\begin{aligned}
n(T)_{850 \mathrm{~nm}}= & 1.75815+1.220 \times 10^{-5} \mathrm{~T}+2.81 \\
& \times 10^{-9} T^{2} .
\end{aligned}
$$

Based on Eqs. (3) and (6), a theoretical prediction of the optical thickness is given as the dotted line in Fig. 7 . The calculation shows a reasonable match to the experimental data.

\section{B. Sensitivity}

Equation (2) indicates that the optical thickness increases with temperature in the form of a convex function. Therefore the sensitivity of the sensor, defined as the slope of the temperature response curve, increases with temperature as well. From Eq. (2) the sensitivity $S$ can be obtained as

$$
\begin{aligned}
S & =\frac{\mathrm{d}}{\mathrm{d} T}[n(T) d(T)] \\
& =1.507 \times 10^{-3}+6.968 \times 10^{-7} T\left(\mu \mathrm{m} /{ }^{\circ} \mathrm{C}\right) .
\end{aligned}
$$



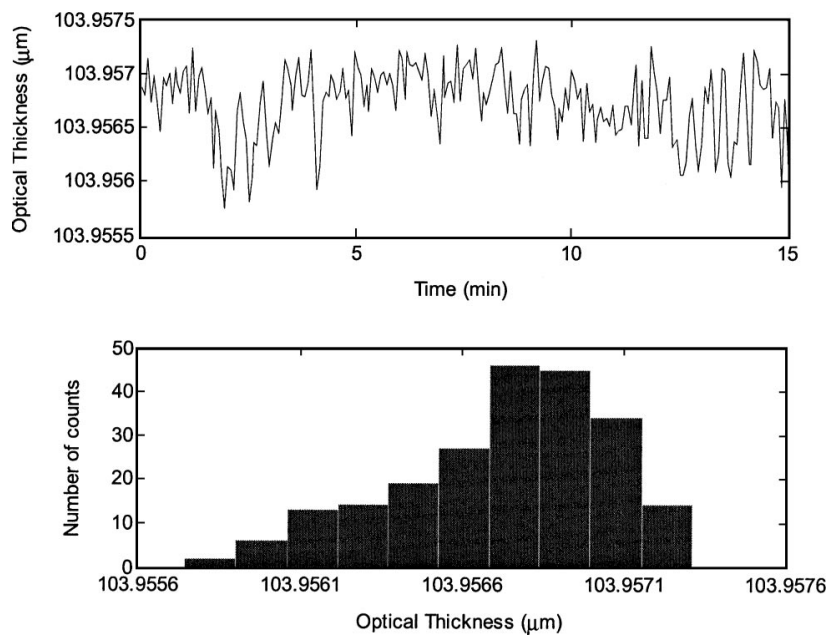

Fig. 8. Sensor resolution at room temperature.

Therefore $S$ increases with temperature from $1.524 \mathrm{~nm} /{ }^{\circ} \mathrm{C}$ at $24{ }^{\circ} \mathrm{C}$ to $2.322 \mathrm{~nm} /{ }^{\circ} \mathrm{C}$ at $1170{ }^{\circ} \mathrm{C}$. Unlike the fluorescence-type sensor whose sensitivities can vary by orders of magnitude over large temperature range, the sensitivities of this sensor remain at a similar level. Hence one can expect similar performance throughout the entire temperature range.

\section{Resolution}

The resolution of the sensor is the smallest temperature change the sensor could resolve. Even under the same temperature, the sensor output (optical thickness) would statistically fluctuate due to system noises. A temperature change is discernible only when it is larger than this fluctuation, to determine which, the sensor's optical thickness was recorded for $15 \mathrm{~min}$. at room temperature $\left(24^{\circ} \mathrm{C}\right)$. Figure 8 shows the result and the statistical distribution. It is then determined that the standard deviation $\delta$ of these data is about $0.3 \mathrm{~nm}$. Therefore the resolution of the sensor at room temperature is

$$
R_{\mathrm{RT}}=\frac{2 \delta}{S_{\mathrm{RT}}}=\frac{2 \times 0.3 \mathrm{~nm}}{1.524 \mathrm{~nm} /{ }^{\circ} \mathrm{C}}=0.4{ }^{\circ} \mathrm{C},
$$

where $2 \delta$ is taken as the smallest discernible temperature change. Better resolution can be achieved by taking the spectrum average. Since $S$ remains at a similar level throughout the temperature range, $R$ will remain at a similar level as well. This means the sensor has a more uniform performance over the entire temperature range and thus a larger dynamic range, as compared to fluorescence-based sensors.

\section{Conclusion}

To conclude, a sapphire-fiber-based extrinsic FabryPerot interferometric sensor has been demonstrated for high-temperature measurement. The sensor is constructed with a $45^{\circ}$-polished fiber end directly bonded to a sapphire wafer to achieve a miniature size by eliminating the need for an extra supporting structure. The adhesive-free coupling of the silica and sapphire fibers is made possible through fusion splicing by the low melting point of the doped silica fiber core, and a loss as low as $0.8 \mathrm{~dB}$ has been measured. Also studied is the dependence of the sensor signal on the alignment angle, showing that a relatively large angle range can be tolerated for high fringe intensity. The prototype sensor was tested from $24^{\circ} \mathrm{C}$ to about $1170{ }^{\circ} \mathrm{C}$ with a resolution of $0.4{ }^{\circ} \mathrm{C}$, and the sensor response is in good agreement with the theoretical prediction.

Besides its reduced size and weight for temperature sensing, the sensor brings the incoming sapphire fiber to the plane of the wafer to achieve a surface mount, offering possibilities for high-temperature structural surface-strain measurements by converting the surface strain to the wafer's thickness change through the Poisson's ratio, which is a fairly efficient 0.3 for sapphire.

This research was sponsored by the U.S. Department of Energy under grant DE-FC26-99FT40685.

\section{References}

1. M. R. Jones, and D. G. Barker, "Use of optical fiber thermometers in high temperature environment," in 11th IEEE International Conference on Advanced Thermal Processing of Semiconductors (IEEE, 2003), pp. 89-100.

2. R. R. Dils, "High-temperature optical fiber thermometer," J. Appl. Phys. 54, 1198-1201 (1983).

3. R. R. Dils, J. Geist, and M. L. Reilly, "Measurement of the silver freezing point with an optical fiber thermometer: proof of concept," J. Appl. Phys. 59, 1005-1012 (1986).

4. L. Tong, Y. Shen, L. Ye, and Z. Ding, "A zirconia singlecrystal fiber-optic sensor for contact measurement of temperatures above $2000{ }^{\circ} \mathrm{C}$," Meas. Sci. Technol. 10, 607-611 (1999).

5. C. W. Meyer, "Effects of extraneous radiation on the performance of lightpipe radiation thermometers," in Proceedings of TEMPMEKO 2001 8th International Zymposium on Temperature and Thermal Measurements in Industry and Science, B. Fellmuth, J. Seidel, and G. Scholz, eds. (IMEKO, 2001), pp. 937-942.

6. D. G. Barker and M. R. Jones, "Temperature measurements using a high-temperature blackbody optical fiber thermometer," J. Heat Transfer 125, 471-477 (2003).

7. J. L. Kenndey and N. Djeu, "Operation of Yb:YAG fiber-optic temperature sensor up to $1600{ }^{\circ} \mathrm{C}$," Sens. Actuators A 100, 187-191 (2002).

8. Z. Zhang, J. H. Herringer, and N. Djeu, "Monolithic crystalline fiber optic temperature sensor," Rev. Sci. Instrum. 68, 2068 2070 (1997).

9. D. M. Henry, J. H. Herringer, and N. Djeu, "Response of $1.6 \mu \mathrm{m}$ Er: $\mathrm{Y}_{3} \mathrm{Al}_{5} \mathrm{O}_{12}$ fiber-optic temperature sensor up to $1520 \mathrm{~K}$," App. Phys. Lett. 74, 3447-3449 (1999).

10. A. Wang, S. Gollapudi, K. A. Murphey, R. G. May, and R. O. Claus, "Sapphire-fiber-based intrinsic Fabry-Perot interferometer," Opt. Lett. 17, 1021-1223 (1992).

11. A. Wang, S. Gollapudi, R. G. May, K. A. Murphey, and R. O. Claus, "Advances in sapphire-fiber-based intrinsic interferometric sensors," Opt. Lett. 17, 1544-1546 (1992).

12. A. Wang, S. Gollapudi, R. G. May, K. A. Murphey, and R. O. Claus, "Sapphire optical fiber-based interferometer for high temperature environmental applications," Smart Mater. Struct. 4, 147-151 (1995).

13. R. E. Wagner and C. R. Sandahl, "Interference effects in optical fiber connections,” Appl. Opt. 21, 1381-1385 (1982). 
14. F. Pérennès, P. C. Beard, and T. N. Mills, "Analysis of a lowfinesse Fabry-Perot sensing interferometer illuminated by a multimode optical fiber," Appl. Opt. 38, 7026-7024 (1999).

15. Y. Zhu, Z. Huang, F. Shen, and A. Wang, "Sapphire-fiber-based white-light interferometric sensor for high-temperature measurements," Opt. Lett. 30, 711-713 (2005).
16. Y. S. Touloukian, R. K. Kirby, R. E. Taylor, and T. Y. R. Lee, Thermal Expansion Nonmetallic Solids, Vol. 13 of Thermophysical Properties of Matter (Plenum, 1977).

17. J. Tapping and M. L. Reilly, "Index of refraction of sapphire between 24 and $1060{ }^{\circ} \mathrm{C}$ for wavelengths of 633 and $799 \mathrm{~nm}$," J. Opt. Soc. Am. A 3, 610-616 (1986). 